\title{
Construcción del proceso de subjetivación en los jóvenes- estudiantes de bachillerato
}

\author{
Construction of the Subjectivation Process in Young High School Students
}

\begin{abstract}
A construção do processo de subjetivação em jovens estudantes do ensino médio
\end{abstract}

Rocío Rodríguez Rico

Universidad Autónoma Chapingo, México

leonorricog@gmail.com

https://orcid.org/0000-0001-9568-6153

Miguel Ángel Samano Rentería

Universidad Autónoma Chapingo, México

masare68@yahoo.com.mx https://orcid.org/0000-0002-8667-9952

Somos sujetos inacabados porque no somos sin otros.

GRACIELA FRIGERIO

\section{Resumen}

Comprender cómo se va gestando el proceso de subjetivación en los jóvenes, de manera particular en aquellos que también desempeñan el papel de estudiantes, y conocer cómo influye el contexto educativo en la construcción de dicha subjetividad es el objetivo primordial de esta investigación. Para ello, se realizaron entrevistas a profundidad con jóvenes-estudiantes de bachillerato. En cuanto a la metodología, se utilizó una del tipo configuracionista. Así, se establece una guía metodológica, pero también ontológica para construir conocimiento. A partir de ella, la realidad es vista en constante transformación y en 


\section{Revista Iberoamericana \\ de las Ciencias Sociales y Humanísticas}

ISSN: $2395-7972$

función de los sujetos. Y se intenta explicar la significación que dan los estudiantes a sus experiencias, no solo como grupo juvenil, sino como personas individuales rodeadas de otras subjetividades que las influyen y las moldean. Se concluye que dicha subjetivación es un proceso complejo que no solo consiste en adquirir un cúmulo de aprendizajes, experiencias propias o ajenas; va más allá de ser sujetos productos de la sociedad que impera y moldea, parte de una lucha constante por deconstruir y construir a partir de sí mismo y los otros, existe una construcción propia a la par de una en colectivo.

Palabras clave: estudiantes, jóvenes, subjetividad, subjetivación.

\section{Abstract}

Understanding how the process of subjectivation is developing in young people, particularly in those who also play the role of students, and knowing how the educational context influences the construction of said subjectivity is the primary objective of this research. To do this, in-depth interviews were conducted with young people-high school students. Regarding the methodology, one of the configurationist type was used. Thus, a methodological guide is established, but also an ontological one to build knowledge. And an attempt is made to explain the significance that students give to their experiences, not only as a youth group, but as individual people surrounded by other subjectivities that influence and shape them. It is concluded that said subjectivation is a complex process that not only consists in acquiring a wealth of learning, own or other people's experiences; it goes beyond being subjects, products of the society that rules and shapes, part of a constant struggle to deconstruct and build from itself and others, there is a construction of its own as well as a collective one.

Keywords: students, young people, subjectivity, subjectivation. 


\section{Resumo}

Compreender como está se desenvolvendo o processo de subjetivação nos jovens, principalmente naqueles que também desempenham o papel de alunos, e saber como o contexto educacional influencia na construção dessa subjetividade é o objetivo principal desta pesquisa. Para isso, foram realizadas entrevistas em profundidade com jovens estudantes do ensino médio. Quanto à metodologia, foi utilizada uma do tipo configuracionista. Assim, estabelece-se um guia metodológico, mas também ontológico para a construção do conhecimento. A partir dela, a realidade é vista em constante transformação e dependendo dos sujeitos. E tenta-se explicar o significado que os alunos atribuem às suas experiências, não apenas como grupo de jovens, mas como indivíduos rodeados de outras subjetividades que os influenciam e moldam. Conclui-se que tal subjetivação é um processo complexo que não consiste apenas em adquirir uma riqueza de aprendizagens, experiências próprias ou alheias; Para além de serem sujeitos, produtos da sociedade que governa e molda, parte de uma luta constante para se desconstruir e construir a partir de si e dos outros, há uma construção própria e também coletiva.

Palavras-chave: estudantes, jovens, subjetividade, subjetivação.

Fecha Recepción: Diciembre 2020

Fecha Aceptación: Junio 2021

\section{Introducción}

Las etapas de evolución por las que transita un ser humano están compuestas de diversos elementos que, de manera ideal, contribuyen a fomentar el desarrollo personal. Al interactuar con personas es importante reconocer las características cognoscitivas, afectivas, volitivas y físicas que las distinguen y diferencian de otras. A partir de estos rasgos los individuos participan de los espacios sociales, abonando tanto en su construcción como en la adecuación de estos. Se trata de la participación social: la adaptación de roles (para efectos de este texto, el de estudiante) y papeles o funciones que toca cumplir (Lomelí, 2009).

A lo largo del desarrollo histórico de nuestra especie se han observado diferentes escenarios de control y dominio. Augusto Comte plantea una subordinación del individuo a la sociedad en términos de sumisión de la libertad individual al orden social y de esta manera visualiza al individuo por primera vez como un producto social (Lomelí, 2009). Cabe señalar 


\section{Revista Iberoamericana \\ de las Ciencias Sociales y Humanísticas}

ISSN: $2395-7972$

que dichos escenarios sociales son influidos a su vez por factores económicos, generacionales y culturales.

Podríamos pensar, entonces, que las personas son construcciones sociales determinadas por las experiencias vividas en cada uno de los espacios en los que se inserta y según las personas y realidades con las que interactúa a través de relaciones dialécticas de interdependencia entre instituciones y los otros.

Si partimos de este pensamiento, cabe preguntarse: ¿la subjetividad del sujeto se construye como el producto de todos los sujetos y experiencias que le anteceden y le van dando forma?

Dicha subjetividad no se reduce solo a la adquisición de cultura, ya que esta se relaciona con el proceso de acumulación social de significados. La subjetividad tiene que ver con la producción de significados a partir de distintos campos subjetivos. En estos campos se realiza la acumulación simbólica de las normas, valores, reglas sociales, intercambios, ideologías, emociones, sentimientos y experiencias, entre otros, que muchas veces dan pie a resistencias y cambios, y reconstruyen así el pasado y a la par construyen un futuro.

Esto nos impulsa a analizar la simbolización, asimilación, adecuación o resistencias que hacen los jóvenes de la cultura dominante que les rodea y les constriñe, pero que no necesariamente les pertenece, y a la cual suman sus experiencias y las de sus pares.

Para acceder a este conocimiento, en primera instancia, se realiza un análisis de la influencia que presentan las instituciones escolares en la construcción de dicha subjetivación, ya que este es un espacio importante de producción y reproducción sociocultural. En este, la subjetividad se desarrolla, reconstruye, expresa y vive. Luego se abre paso al análisis del proceso de subjetivación, y de manera particular la construcción que se hace de este desde la perspectiva de los jóvenes-estudiantes, perspectiva que difiere de otros jóvenes quienes se encuentran en contextos y realidades distintas.

\section{Metodología}

Para abordar esta investigación recurrimos a una metodología configuracionista, ya que fuimos construyendo nuestro objeto de estudio a través de las perspectivas de los jóvenes, de su presente y las expectativas hacia el futuro. Se trata de entender la realidad en movimiento y en constante contradicción, de observar a los jóvenes no desde el papel que deben desempeñar, solo como actores en representación, sino desde el que viven, de manera 
activa, cuestionadora y constructiva. Los datos cualitativos obtenidos fueron aplicados a un estudio de caso intrínseco, de tipo descriptivo. Así, se subrayan aspectos relacionales y se identifican de manera analítica puntos de contacto, todo ello gracias a entrevistas a profundidad realizadas a alumnos de bachillerato.

\section{Discusión}

\section{La influencia de las instituciones escolares...}

Las instituciones escolares procuran desarrollar en los jóvenes habitus en torno al conocimiento, la ciencia, la racionalidad, la tecnología y la cultura. Se estructura así un sistema jerárquico y de prestigios en el que se reconoce como valor dominante el saber. En muchas de estas instituciones se promueve cada vez menos un carácter social y compartido del aprendizaje y, en cambio, se le da una mayor relevancia a las políticas de competitividad, a la desvalorización de lo humano y al desarraigo del hombre. En muchos de estos contextos áulicos aún se respira una lucha del poder y una disputa por ser "el más apto", catalogado así a través de diversas prácticas (Bethencourt y Villegas, 2011, p. 153).

Uno de los retos importantes que hasta hoy no se ha logrado del todo es fomentar en los jóvenes una educación integral; situación, la de la ausencia de la educación integral, que, entre otras cosas, lleva a la educación a los resultados que actualmente se gestan. Para cambiar esto, se requiere una revisión en los planes y programas de estudio (Gómez, 2017), adecuarlos a los requerimientos de quienes los viven, pues pareciera que se intenta ignorar a los actores primordiales de los recintos escolares en favor de una esclavización tecnoindustrial.

Se privilegian los cálculos y los datos exactos, se homogenizan los conocimientos y se trata de generar estandarización, se desestima la individualidad y se soslaya la esencia humana. En suma, se desarrollan personas productivas y se instaura la razón como fuerza ordenadora, pero no la racionalidad o el desarrollo del pensamiento crítico. Ser diferente es cuestionado y algunas veces incluso castigado (Bethencourt y Villegas, 2011).

Este tipo de prácticas generan hábitos de consumo y necesidades diversas que benefician y promueven la expansión industrial. De esta manera, se invisibiliza a los sujetos y se percibe a estos solo como partes de una estructura o un engranaje con objetivos mayores y de mayor importancia que el que puedan manifestar los jóvenes estudiantes. En la cultura de la modernidad, se favorece al individuo desarticulado. Los componentes de su esencia son 


\section{Revista Iberoamericana de las Ciencias Sociales y Humanísticas}

ISSN: $2395-7972$

desagregados y, por extensión, se convierte en un sujeto más proclive a la alienación y la reproducción. La modernidad es indiferente a las necesidades subjetivas y humanas de la persona diversa e inacabada que constantemente se está formando (Bethencourt y Villegas, 2011, p. 152).

Entonces, ¿cómo pueden los espacios educativos relacionarse con los jóvenesestudiantes? ¿De qué manera se pueden desarrollar vínculos entre las demandas escolares y las demandas de quienes asisten a estos recintos?

A partir de la reforma educativa de 2013, el nivel de educación medio superior se planteó como obligatorio con la finalidad de profundizar en la formación integral de los estudiantes con independencia de que puedan continuar su educación superior o se integren al mundo laboral (Plan Nacional de Desarrollo 2013-2018, 20 de mayo de 2013).

En la educación actual, es importante instaurar un cambio fundamental; es necesario recurrir a la transversalidad; es decir, desarrollar las capacidades y habilidades integrales de cada ciudadano en los ámbitos intelectual, afectivo, artístico y deportivo. Paralelamente, resaltar valores a través de los cuales se reconozca la dignidad propia y la de otros, fomentando un cambio en la actitud del estudiante, orientarlo hacia la convivencia, la tolerancia y la formación de una conciencia personal (Gómez, 2017).

Bethencourt y Villegas (2011) citan a varios autores que resaltan a "la convivencia, elemento substancial del homo socialis; en esto subyace la creencia de acuerdo con la cual el entendimiento del ser humano solo será posible cuando se piense en él desde la relación antropológica individuo-especie-sociedad" (p. 154). Esta trinidad es determinante para la construcción subjetiva de las realidades vividas por los sujetos.

\section{Subjetividad}

Si bien la subjetividad inicia siendo un debate teórico y epistemológico de la filosofía, a partir de la década de 1960 se vuelve un tema central en el análisis social y comienza a ser visto desde diferentes vertientes, ya que a través de estas subjetividades se pueden gestar cambios políticos, económicos, sociales, culturales y educativos (Aquino, 2013).

Pensadores como Karl Marx, Max Weber y Émile Durkheim, quienes sientan las bases para nuestra comprensión del orden social e identifican las relaciones sociales de producción, la división del trabajo social y la dominación política como condiciones de 


\section{Revista Iberoamericana \\ de las Ciencias Sociales y Humanísticas}

posibilidad de orden, se percatan que estas estructuras operan sobre las espaldas de los sujetos y no pueden reproducirse sin su relativo consentimiento e involucramiento.

Bajo la visión de Marx, el ser humano plasma su subjetividad en el trabajo y de esta manera se objetiva en un producto material; tanto los medios de producción como el producto del trabajo se subordinan a la producción capitalista, lo que lleva al ser humano a una enajenación. Marx no describe procesos de subjetivación exitosos, ya sea en términos de toma de conciencia o de desalienación, pero presenta a la subjetividad como un límite a la producción del sistema y como la base de su transformación (Angelcos, 2017).

Para Weber, la racionalización no implica solamente una mayor autonomía de los sujetos respecto a la tradición, sino también la automatización creciente de la acción racional. Así, las personas reconstruyen el mundo en la intimidad de su experiencia individual, lo que da un poco de luz a la subjetividad.

Durkheim, por su parte, considera que el rol de la persona dentro de la división social es una condición motivacional suficiente para mantener la integración social, en el sentido de reproducir la tradición cultural que ha sido transmitida por la socialización, ya que cuando los individuos actúan reproducen el rol que ocupan dentro de la estructura social. Por lo que las instituciones educativas permiten interiorizar normas sociales dentro de la conciencia de los individuos y sancionar de manera tanto formal como informal las conductas desviadas (Angelcos, 2017).

En resumen, los conceptos de alienación en Marx, de pérdida de sentido y libertad en Weber y de anomia en Durkheim hacen referencia a aquella dimensión de la acción que no sería integrada de forma adecuada en la reproducción del orden social. Dicha distinción sentó las bases para pensadores posteriores que dan un mayor abordaje al término subjetividad.

En esa línea, el sociólogo Alain Touraine argumenta que existe un distanciamiento crítico experimentado por los individuos según los roles que la sociedad les impone a partir de una cultura dominante, lo que lleva a que los individuos busquen regular su deseo más allá de lo prescrito por las orientaciones culturales dominantes y da origen a la posibilidad del desarrollo histórico-contingente de la sociedad (Aquino, 2013).

Por su parte, Michael Foucault (2002) concibe al sujeto como producto del discurso en dos diferentes sentidos: sujetos que personifican las formas particulares de conocimiento que el discurso produce y como lugares para el sujeto (posiciones subjetivas). De ahí que 


\section{Revista Iberoamericana de las Ciencias Sociales y Humanísticas}

ISSN: $2395-7972$

todos los individuos, en un periodo determinado, lleguen a ser sujetos de un discurso particular y, por lo tanto, el sujeto se convierte en un efecto del poder y en el resultado de un conjunto de técnicas, ciencias y otros tipos de dispositivos que permiten la fabricación del "individuo disciplinario" (Foucault, 2002)

Desde esta perspectiva, la subjetividad es resultado de los mecanismos de normalización en el individuo, es decir, de la forma en que los dispositivos disciplinarios se articulan entre sí y producen un tipo de mentalidad congruente con las condiciones culturales existentes.

Por otro lado, Felix Guattari (1992) señala que la subjetividad se manufactura como la energía, la electricidad o el aluminio; de ahí que la producción de subjetividad posiblemente sea más importante que cualquier otro tipo de producción. ¿Cómo se produce la subjetividad desde esta perspectiva? A partir de los medios de comunicación de masas, la publicidad, los sondeos, las estadísticas, las encuestas, que fabrican la opinión a gran escala y crean actitudes estereotipadas y narrativas de deseo escleróticas. Se trata de sistemas de conexión directa entre las grandes máquinas productivas, las de control social y las instancias psíquicas que definen la manera de percibir el mundo.

No hay sujeto sino subjetividad, o, mejor dicho, subjetividades. En efecto, Guattari concibe a la subjetividad como múltiple, dispar, fragmentada, heterogénea, como si existieran tantas subjetividades como situaciones y momentos; sin embargo, la subjetividad es, al mismo tiempo, una: la subjetividad capitalista (Aquino, 2013).

Gran parte de la subjetividad del sujeto parte de la cultura, ya que las acciones, opiniones, actitudes no pertenecen al sujeto propiamente dicho, son mediadas por estructuras internalizadas, un habitus, es decir, un sistema de disposiciones que inclina a los actores a actuar, pensar, sentir y dar sentido de una manera coherente con la estructura en la que socializan (Bourdieu, 2007).

Para Touraine (1997), definirse como sujeto implica la capacidad para reflexionar sobre sí mismo, reconocerse en la vida que cada uno controla pero que al mismo tiempo nos es impuesta por nacimiento. Ser creador de sentido y de cambio, de relaciones sociales e instituciones políticas, ser actor de su propia vida y, en este proceso, transformar a la sociedad, muchas de las veces sin darse cuenta.

Asimismo, Araujo y Martuccelli (2010) argumentan que los individuos siguen siendo socializados a través de factores culturales, pero esta socialización opera en un contexto en 


\section{Revista Iberoamericana \\ de las Ciencias Sociales y Humanísticas}

ISSN: 2395 - 7972

el cual la cultura posee cada vez un rol más ambivalente y cada individuo se vuelve el fruto de una serie cada vez más contingente y diversa de experiencias. Se impone la necesidad de reconocer la singularización de las trayectorias personales. Lo que proponen Araujo y Martuccelli (2010) es analizar la producción de individuos — la individuación — a partir de algo que llama pruebas, es decir, desafíos históricos, socialmente producidos, culturalmente representados, desigualmente distribuidos que los individuos están obligados a enfrentar en el seno de un proceso estructural de individuación.

En épocas y contextos heterogéneos, estos autores han abordado la subjetividad como un elemento de control, de reproducción o, en el polo opuesto, como un elemento a través del cual emanan procesos emancipatorios de las culturas dominantes. Lo cierto es que todos ellos otorgan una importancia relevante a esta para gestar cambios diversos, ya sea desde la reproducción o como camino a la liberación y evolución.

\section{Proceso de subjetivación}

La construcción de la subjetivación se da en diversos campos de interacción. Las relaciones en el aula son también una construcción subjetivamente humana en la cual los encuentros entre el yo y los otros siempre serán deseables y nutrientes. La construcción de las personas se da mediante un proceso continuo que no se realiza en solitario, es decir, se forma en conjunto por diversos actores, instituciones y la cultura. Esta se recrea a sí misma en cada sujeto y cada sujeto da cuenta de ella. Es así como las subjetividades colectivas proponen los ejes a partir de los cuales el sujeto se construye desde lo simbólico, a la par de significaciones sociales personales y colectivas (Lomelí, 2009).

El proceso de subjetivación se caracteriza en estos jóvenes-estudiantes por el distanciamiento de normas y valores, el desarrollo de gustos e intereses específicos, el conocimiento y desarrollo de capacidades propias, la reflexión y la toma de decisiones, que muchas de las ocasiones distará de la impuesta por el grupo de adultos que los rodea. Este se ve acompañado e influenciado de manera importante por el cúmulo de experiencias, los otros, las prácticas de sus pares y las experiencias de estos. De esta manera se logran desarrollar las identidades (Weiss, 2012).

La subjetividad es el producto específico de múltiples modos de subjetivación y procesos dialógicos, el espacio de construcción de cada sujeto, construcción realizada desde lo colectivo, pero de manera individual. Así, se origina de la asimilación de las diversas 


\section{Revista Iberoamericana \\ de las Ciencias Sociales y Humanísticas}

ISSN: $2395-7972$

experiencias, personas e instituciones que dan origen a dicho sujeto. Y a través de este, del sujeto, se muestra la generalidad, especificidad, singularidad, diversidad y su historia.

\section{Los jóvenes y su subjetividad}

Taguenca (2009) define a los jóvenes como personas en busca de una identidad propia. Y aclara que dicha identidad se construye desde una heterogeneidad no carente de complejidad. Con proyectos diferenciados los jóvenes se insertan en diferentes instituciones educativas que se encuentran normadas por la sociedad dominante, la de los adultos. En esa línea, el docente propone un abordaje teórico de transmisión de contenidos encaminado a desarrollar saberes específicos y de carácter escolarizante, esto es, orientado al disciplinamiento.

El proceso de subjetivación no es el mismo para los jóvenes que asisten a la escuela que para quienes no lo hacen o abandonan dicho trayecto. Foucault (1988) menciona: “El poder clasifica a los individuos, los jerarquiza, los designa y les impone una identidad (como acepción 'verdadera' de sí) en la que deben reconocerse y ser reconocidos. Así el poder transforma a los individuos en sujetos" (p. 231). La subjetivación se da entonces como un proceso de tensión en el cual los individuos tienen una sujeción; al mismo tiempo, a través de este mismo proceso, logran cierta libertad. Se logra una creación de sí mismo, se genera un sujeto capaz de actuar, con voluntad propia, desarrollada en sociedad, pero individual, con cierta libertad para tomar decisiones y conocer que existen consecuentes: un sujeto con capacidad de agencia.

La subjetividad es el devenir siempre abierto al proceso de subjetivación. En consecuencia, la formación se realiza a través del proceso de subjetivación donde el sujeto se transforma adquiriendo y/o cambiando capacidades, formas de sentir, de actuar, de imaginar, de comprender, de aprender (Ferry, 1990, p. 52, citado en Anzaldúa, 2009, p. 7).

Al transitar por la adolescencia se va construyendo un sí mismo diferente al que se ha sido hasta ese momento, con demandas, confusiones e inquietudes propias de la etapa juvenil que se vive. Se desarrolla una vida colectiva independiente de la vida escolar, una vida subjetiva que no puede desarrollarse separada de la vida escolar, ya que afectará a esta en diferentes momentos (Weiss, 2012). 


\section{Revista Iberoamericana de las Ciencias Sociales y Humanísticas}

ISSN: $2395-7972$

Al no existir una separación determinante entre los ámbitos juveniles y la vida escolar en los jóvenes-estudiantes, cobra importancia trazar puentes analíticos y de investigación entre el campo educativo y el de la juventud, pues se asume, de entrada, que la escuela no es un espacio social, se tiene la idea de que los sujetos solo la viven como estudiantes, dejando de lado que, a la par de ser estudiantes, son jóvenes en proceso de construcción. Ambas condiciones construyen de manera distinta sus ideales, sus relaciones y, con ello, el proceso de subjetivación (Weiss, 2012). A los jóvenes-estudiantes se les concibe como receptores de normas, principios, cultura, información, entre otros.

Al respecto, Dubet y Martuccelli:

Enfocan en paralelo el proceso de socialización y de individualización y afirman que la experiencia se desarrolla en tres lógicas de interacción: la interiorización de normas o roles (socialización), el desarrollo de una subjetividad personal en forma de gustos e intereses que conduce a los individuos a establecer una distancia con su socialización (subjetivación) y la actuación instrumental o estratégica, cifrada en un cálculo de utilidad de inversión en las tareas escolares con vistas a proyectos futuros y considerando sus recursos y recorrido escolar previo (estrategia) (Weiss, 2012, p. 136).

Estos procesos se van realizando a la par de que el estudiante transita por los diferentes niveles académicos en los que se inserta. Así pues, en primera instancia, se socializa con base en las normas transmitidas intergeneracionalmente y con base en su interiorización; se da un proceso de inserción en los esquemas cognitivos y morales del individuo y su consecuente modificación. En palabras de Piaget, un "proceso de asimilación y acomodación". Posteriormente, se vive un proceso conflictivo, pues las acciones, pensamientos y emociones de los sujetos funcionan de forma flexible; su proceder no se limita solo al seguimiento de esquemas, procedimientos y reglas; existe reinterpretación y adecuación. Por lo tanto, el estudiante desarrolla su subjetividad para llegar a tomar una postura estratégica que le acerque de manera eficiente a sus metas y objetivos propios, a través de una transformación, reformulación y actuación con decisiones basadas en criterios morales propios, es decir, se hace responsable de sí mismo.

En las sociedades actuales, la educación escolarizada ocupa un espacio central en el proceso de socialización y formación de los jóvenes. Desde una perspectiva crítica, Bordieu y Passeron (1996) enfatizaron la función reproductora de la socialización y la reproducción 


\section{Revista Iberoamericana de las Ciencias Sociales y Humanísticas}

ISSN: 2395 - 7972

de la sociedad de clases por la escuela. Sin duda alguna, los estudiantes que se encuentran en bachillerato han socializado de manera exitosa el oficio de ser estudiantes y lo que ello conlleva. Los jóvenes que asisten y permanecen en el sistema educativo obtienen la denominación de estudiantes y con ello un rol social claramente determinado, y con cierto valor e importancia tanto personal como socialmente, esto independientemente del valor de los aprendizajes que se adquieren. Aquellos que no logran adaptarse a esta situación abandonan este nivel en los primeros semestres (Camarena, 2000).

Los adolescentes y jóvenes tienen un mundo separado de los adultos, a pesar de compartir el espacio áulico, y es en este mismo espacio donde se desarrollan valores, reglas y jerarquías propias. En la mayoría de estos, existe un nítido interés por la "sociabilidad". Simmel (2002) enfatiza que es el gusto de la unión con otros y el aspecto lúdico de estar juntos. En paralelo al proceso de socialización, se desarrolla el proceso de subjetivación.

Al desestimar u obviar la experiencia juvenil en los espacios académicos, pareciera que se omite que la escuela es también un espacio de vida juvenil, y que la experiencia escolar tiene peso en la construcción de las varias identidades de los sujetos tanto dentro como fuera del espacio escolar (de Garay, 2013).

Debido a nuestra constitución social, este espacio se convierte en un lugar primordial para el desarrollo de la subjetividad. Según Martuccelli (2007), el concepto de subjetivación enfatiza más la emancipación de estructuras y valores dominantes y la elaboración de normas y valores propios. Dicha subjetividad se expresa y puede ser observada, no desde la introspección, sino desde el actuar de las personas, en sus gustos, intereses, en las prácticas que realizan, en la manera de expresarse sobre quienes y lo que les rodea y sobre sí mismas. Se observa en la singularidad de cada individuo y en el desarrollo del humano en los diferentes espacios de formación.

Es por esta misma subjetividad que al insertarse en los espacios académicos no existen trayectorias lineales ni homogéneas, no hay rutas normales a seguir, menos las establecidas por las instituciones académicas. A pesar de que los sistemas sociopolíticos erigen dominios que emiten e institucionalizan discursos de poder y jerarquizan las diferencias, las subjetividades colectivas brindan la cultura a cada ser que nace y lo dotan de una forma única e inigualable de leer la realidad, de aprenderla y transformarla desde sus propias experiencias. A partir de conocimientos propios se producen imaginarios (Lomelí, 2009). 


\section{Revista Iberoamericana \\ de las Ciencias Sociales y Humanísticas}

ISSN: $2395-7972$

Al estar inmersos en este espacio los jóvenes traen consigo su mundo, ideas y prácticas encaminadas a romper las reglas y transgredir los límites impuestos por los adultos, ir contra lo convencional y lo preestablecido, cuestionar lo que inculcan los padres y docentes en la idea de crear y recrear espacios haciendo usos estratégicos de las reglas para lograr así sus objetivos, y lograr identidades propias, mantener charlas sobre lo vivido, sexo, amor, intereses, familia, conflictos, preocupaciones, entre otros (de Garay, 2013).

La condición de ser joven y estudiante a la vez no se contrapone, sin embargo, debe tener cabida esta juventud en los recintos educativos y a la par entender que la escuela no es el único espacio formativo. En la actualidad, los jóvenes se encuentran expuestos de manera constante e inagotable a información diversa que puede desarrollar aprendizajes o no, pero que no deja de tener importancia en sus diversas construcciones.

Se desarrolla así una tensión que muchas veces no se logra suavizar entre los códigos de las instituciones educativas, que generalmente son rígidos, fríos y estáticos, y, por otra parte, los flexibles, veloces y desestructurados con los que se desenvuelven los medios de comunicación y que empatan de una manera más atractiva con las características tanto de pensamiento como de conducta con los jóvenes, quienes, en muchas ocasiones, son seducidos por estos (de Garay, 2013).

Podríamos cuestionar, entonces, tomando en cuenta que este proceso de subjetivación se desarrolla de manera intensa entre la socialización gestada en los grupos juveniles y que la adquisición del conocimiento se puede dar de manera independiente a través de las diversas tecnologías de la información y la comunicación (TIC), ¿para qué sirven los docentes y la cultura académica?

En los espacios escolares se gesta de manera particular un proceso de construcción, no solo una construcción basada en los conocimientos o en las prácticas sociales entre pares y docentes; en este espacio la construcción va más allá, se desarrollan habilidades específicas, se conocen y discuten normas. Se enfrenta a los jóvenes a experiencias constructivas que solo se asimilan en lo colectivo y se representan en lo individual, retomando en esta construcción lo propuesto por Herder. 


\section{Revista Iberoamericana de las Ciencias Sociales y Humanísticas}

Herder, una de las figuras centrales del romanticismo alemán, proclamó a inicios del siglo XIX — en contra de la simple transmisión de conocimientos y buenas maneras, que llamó educación-, el concepto de bildung como un proceso para hacer a las personas capaces de pensar, juzgar y actuar por sí mismas, un proceso que requiere "la actuación viva del enseñante y la actividad del que se forma" (Roldán, s. f.). Bildung significaba también la totalidad de las experiencias que permiten desarrollar una identidad coherente, no solo en términos individuales sino también colectivos (Weiss, 2014, p. 9).

El transitar de los jóvenes por los espacios áulicos va construyendo identidades únicas y diferentes. No solo atraviesan un proceso formativo a nivel cognitivo, sino que son partícipes de procesos de mayor importancia, como aquellos que dan estructura y forma a sus objetivos y metas, tanto en lo individual como en lo colectivo. Al pensar en el término subjetivación como un proceso que comprende el desarrollo de ideas, criterios y gustos propios; la apropiación de flujos culturales, la emancipación de normas y valores dominantes; el conocimiento emocional de sí mismo y la capacidad de reflexionar y decidir; al concebirlo de esta forma, decíamos, se denota una alta coincidencia con el concepto de formación, pues se trata de un proceso de desarrollo de capacidades a través de una relación intersubjetiva, el reconocimiento del otro o los otros como sujetos, aun en la desigualdad, y una apropiación de la cultura, como una persona responsable capaz de cuidarse a sí mismo y también a los otros. Como cita Hegel, "la conciencia de sí del sujeto se transforma en conciencia para sí”. Si logro ver en el otro un sujeto, con sus diferencias y vicisitudes particulares, si logro ver al otro como sujeto, soy capaz de ver el sujeto en mí. Entonces, el proceso de diálogo entre el estudiante y el docente se presenta como una lucha por el reconocimiento y un retorno sobre sí mismo.

Una de las preocupaciones en los recintos educativos es cómo desarrollar formas eficaces para que los jóvenes no deserten de los espacios académicos, y provocar apasionamiento en estos por el conocimiento. Una respuesta puede ser proponer a los jóvenes situaciones menos escolares y más significativas en términos de vida; proponer situaciones a través de las cuales en verdad desarrollen procesos de aprendizaje. No se trata de renunciar a la cultura académica, sino de lograr su apropiación mediante una diálogo que permita mediar entre el horizonte académico y el horizonte juvenil (Weiss, 2014). 


\section{Resultados}

Al intentar cumplir con la demanda de proporcionar educación a todos los jóvenesestudiantes se realiza un proceso de masificación y homogeneización del conocimiento. Lo anterior, sin embargo, al paso de los años, resulta ser una solución poco eficiente y de alcances mínimos que atenta contra la individualidad de los estudiantes, ya que estos asisten a las aulas sin un conocimiento pleno y definido de sus proyectos de vida o expectativas individuales concretas o en construcción. Algunos de ellos externan: "Quiero estudiar para acceder a una mejor calidad de vida", o bien: "Si logro terminar la preparatoria, seré alguien", o bien: "En esta ocasión haré de todo para no abandonar la escuela otra vez, esta es la manera de ser mejor".

Dentro de los contextos áulicos se visualizan diferentes mundos, percepciones e intereses no compartidos, pero que tampoco son rechazados completamente. Se genera una adaptación a través de la reinterpretación. "No me gusta mucho hacer amigos, muchas de las veces hablan de tonterías, pero después hablamos de cosas serias, como de las materias y eso". "Los chavos de mi grupo son raros, pero aun así formamos grupos de trabajo, algunos aportan ideas que nos ayudan a todos"

Dicho proceso de subjetivación se desarrolla durante el transitar de los sujetos por su historia y en la construcción también de la historia social a través de su inserción en diversas instituciones, las cuales van abonando a la construcción de la identidad o de la autoría del yo.

En este contexto, asimilan e integran reglas, normas y lineamientos impuestos por dichas instituciones de las que ellos forman parte, muchas veces por decisión y otras por circunstancias diversas. Por ello, en ocasiones los estudiantes intentan aislarse en sus propias subjetividades y la asistencia a las clases se vive desde el "deber". "Las clases son aburridas, pero si no asisto me reprueban". "La verdad prefiero trabajar y a clases voy pocas veces, pero entrego las actividades".

Un distintivo de los jóvenes-estudiantes es justamente que se dedica una parte importante de su tiempo a mantener una relación con el espacio institucional, perfectamente establecido y normado. La escuela se convierte en un espacio relevante en sus vidas, pero no solo con cortes o tintes desde los elementos académicos, sino también como espacio de socialización, donde se viven tanto encuentros como desencuentros. Los jóvenes estudiantes viven en y para la escuela. "Cada día me levanto, me alisto y tomo clases, desayuno y después 


\section{Revista Iberoamericana \\ de las Ciencias Sociales y Humanísticas}

ISSN: 2395 - 7972

realizo tareas, investigaciones y esas cosas de la escuela. Cuando termino, casi es hora de dormir".

En estos espacios áulicos, los jóvenes no solo viven aventuras, también reflexionan, aprenden de sus experiencias, se conocen mejor a sí mismos y trazan caminos y proyectos, aprenden a hacerse cargo de su vida, que muchas veces se vuelve incierta y sin una trayectoria ni laboral ni académica trazada, se esbozan proyectos temporales

Debido a esta subjetividad es que hay que conocer el mundo de la vida juvenil de los estudiantes, porque este está plagado de experiencias personales, familiares, culturales, gremiales (a la par de las educativas). Esto lleva a los jóvenes-estudiantes a vivir procesos de transición múltiples, bañados con la euforia de este periodo de la vida. Los tiempos establecidos por los jóvenes no son los mismos de los adultos y mucho menos se empatan con los de las instituciones y sus necesidades de cubrir estándares de calidad. "Esta es la tercera vez que intento terminar la preparatoria, he tenido muchos problemas". "En casa vivimos mi mamá y mi hermana, pero mi mamá perdió su trabajo, ahora vendemos postres y con eso pagamos los gastos".

\section{Conclusiones}

En la subjetivación de los jóvenes estudiantes se entrecruzan diferentes temas y esferas de desarrollo, una gran diversidad de vivencias atraviesa el proceso, ya que existen diversas formas de simbolizar el tránsito por los recintos educativos, temas como vivir la preparatoria, lograr un ingreso a la universidad o al campo laboral; ver la vida escolar como un medio para superar condiciones de desigualdad de género, conseguir prestigio y ser reconocido socialmente o para lograr una movilidad social y económica; adquirir autoestima y valoración social y personal.

En cuanto al imaginario, es una creación constante, indeterminada de figuras, formas e imágenes, así como una creación social, histórica y psíquica. Nuestra construcción subjetiva incluirá normas, valores, lenguaje, herramientas, métodos, pensamientos de los otros que estarán encaminados a construir al individuo mismo e irrepetible. Es un proceso constante de tensión entre significaciones sociales e individuales, entre lo ya hecho y lo que se está construyendo. 


\section{Revista Iberoamericana \\ de las Ciencias Sociales y Humanísticas}

ISSN: $2395-7972$

Y es justo por esta construcción que adquiere una importancia especial mirar la subjetividad en los recintos educativos, porque es a través de esta que se generan cambios políticos, económicos, sociales, culturales y educativos, no solo para los jóvenes, sino para la sociedad en general, ya sea desde la reproducción de las culturas dominantes o desde la búsqueda de la emancipación.

La construcción de la subjetividad se va desarrollando por diversas etapas. En los primeros años de escolarización se da un proceso de socialización y, con ello, la asimilación de normas, reglas y maneras de actuar, pero conforme el estudiante se vuelve más reflexivo comienza a reconocer su lugar en la sociedad, la cultura y en la vida en general, se da una reinterpretación y adecuación del contexto, de sus objetivos y de hacia dónde pretende dirigirse. Muchas de las ocasiones el camino decidido no es el impuesto por los adultos.

En el discurso de los jóvenes se puede ver la interiorización de normas e ideas y preceptos de la cultura de las generaciones que les anteceden. Ambos, ideas y preceptos, entran en conflicto con los nuevos conocimientos, experiencias y panoramas que van adquiriendo a través de la interacción en los espacios áulicos. Al ser la escuela una transmisora de las normas de la sociedad y vivir en ella también una cultura juvenil, se da un proceso de conflicto, asimilación y negociación que llevará al joven a desarrollar y formar su subjetividad.

He aquí la importancia de realizar procesos de construcción sólidos, con base en valores grupales y con objetivos y metas claras, para instar el desarrollo de una subjetividad que encamine la transformación social, transformación que nos lleve a ser una sociedad para sí.

Como reflexión final, y a modo de respuesta a la pregunta inicial, a saber, "¿la subjetividad del sujeto se construye como el producto de todos los sujetos y experiencias que le anteceden y le van dando forma?", podríamos decir que la subjetividad del sujeto se construye en lo colectivo, en lo cultural, en lo social, en lo económico y político, pues en el sujeto se cristaliza un colofón de aquellos momentos y experiencias que le construyen, sin embargo, no se desarrolla una subjetividad universal, porque esta interactúa también con las particularidades de los sujetos. Así, a pesar de que existe una generación de jóvenes expuestos a una gama de experiencias sociohistóricas similares, su comportamiento puede distar de manera importante. 


\section{Revista Iberoamericana \\ de las Ciencias Sociales y Humanísticas}

ISSN: $2395-7972$

Es por eso por lo que los espacios escolares se vuelven importantes, ya que siguen siendo el trayecto designado para la mayoría de los jóvenes y es en este donde pasan la mayor cantidad de su tiempo. Se puede, pues, abonar al proceso de subjetivación gestando espacios de reflexión y de acción para desarrollar una agencia del yo y, a través de ella, llevar a cabo actividades propias, encaminadas a lograr objetivos en lo particular que acerquen al sujeto a la consecución de diversas metas, a través de utilizar recursos simbólicos de la cultura para construir una identidad y las prácticas que le acompañan.

Como línea de seguimiento nos quedamos con las interrogantes sobre cómo cambia esta construcción de la subjetividad en los jóvenes estudiantes en un proceso educativo a distancia, el cual, actualmente, se vive no por elección, sino por necesidad debido a la pandemia por la enfermedad por coronavirus de 2019 (covid-19). ¿Cambia el proceso de subjetivación? ¿Se construye con otros elementos? ¿Este cambio en la socialización da origen a otras subjetividades?

\section{Referencias}

Angelcos, N. (2017). Subjetividad, cuerpo y afecto en la teoría sociológica. Revista Estudios Avanzados, (26), 76-94.

Anzaldúa, R. E. (2009). La formación: una mirada desde el sujeto. Ponencia presentada en el X Congreso Nacional de Investigación Educativa. Veracruz, del 21 al 25 de septiembre de 2009. Recuperado de http://www.comie.org.mx/congreso/memoriaelectronica/v10/pdf/area_tematica_15/ ponencias/0251-F.pdf.

Aquino, A. (2013). La subjetividad a debate. Sociológica, 28(80), 259-278.

Araujo, K. y Martuccelli, D. (2010). La individuación y el trabajo de los individuos. Educação e Pesquisa, 36, 77-91.

Bethencourt, M. T. y Villegas, M. M. (2011). El alumno humano adolescente. Una lectura desde el aula de educación media. Revista Paradigma, 32(2), 151-168.

Bourdieu, P. (2007). El sentido práctico. Buenos Aires, Argentina: Siglo XXI Editores.

Bourdieu, P. y Passeron, J. C. (1996). La reproducción: elementos para una teoría del sistema de enseñanza (2. ${ }^{a}$ ed.). Ciudad de México, México: Fontamara.

Camarena, R. M. (2000). Los jóvenes y la educación: situación actual y cambios intergeneracionales. Papeles de Población, 6(26), 25-41. 
de Garay, A. 2013. El mundo juvenil de los estudiantes de bachillerato. Revista Mexicana de Investigación Educativa, 18(57), 637-643.

de la Garza, E. y Leyva, G. (coords.) (2012). Tratado de metodología de las ciencias sociales. Perspectivas actuales. Ciudad de México, México: Universidad Autónoma Metropolitana-Fondo de Cultura Económica.

Dubet, G. y Martuccelli, D. (1998). En la escuela. Sociología de la experiencia escolar (1. ${ }^{a}$ ed). España: Losada.

Foucault, M. (1988). El sujeto y el poder. Revista Mexicana de Sociología, 50(3), 3-20.

Foucault, M. (2002). Vigilar y castigar (1. ${ }^{\mathrm{a}}$ ed.). Buenos Aires, Argentina: Siglo XXI Editores.

Gómez, M. E. (2017). Panorama del sistema educativo mexicano desde la perspectiva de las políticas públicas. Innovación Educativa, 17(74), 1665-2673.

Guattari, F. (1992). Chaosmose. Paris, France: Galilée.

Lomelí, L. (2009). Tras las huellas de la derecha. Renglones.

Plan Nacional de Desarrollo 2013-2018. (20 de mayo de 2013). Diario Oficial de la Federación. Recuperado de http://www.dof.gob.mx/nota_detalle.php?\%20 codigo $=5299465 \&$ fecha $=20 / 05 / 2013$.

Simmel, G. (2002). Cuestiones fundamentales de sociología. Barcelona, España: Gedisa.

Taguenca, J. A. (2009). El concepto de juventud. Revista Mexicana de Sociología, 71(1), 159-190.

Touraine, A. (1997). ¿Podremos vivir juntos? Iguales y diferentes. Ciudad de México, México: Fondo de Cultura Económica.

Weiss, E. (2012). Los estudiantes como jóvenes: el proceso de subjetivación. Perfiles Educativos, 34(135), 134-148.

Weiss, E. (2014). Subjetivación y formación de la persona. Ponencia presentada en el Congreso Internacional AFIRSE. Ciudad de México, del 17 al 20 de junio de 2014. 
Revista Iberoamericana

de las Ciencias Sociales y

Humanísticas

ISSN: 2395 - 7972

\begin{tabular}{|l|l|}
\hline Rol de Contribución & Autor (es) \\
\hline Conceptualización & Rocío Rodríguez Rico \\
\hline Metodología & Rocío Rodríguez Rico \\
\hline Software & Rocío Rodríguez Rico \\
\hline Validación & Rocío Rodríguez Rico \\
\hline Análisis Formal & Rocío Rodríguez Rico \\
\hline Investigación & Rocío Rodríguez Rico \\
\hline Recursos & Rocío Rodríguez Rico \\
\hline Curación de datos & Miguel Ángel Samano Rentería \\
\hline $\begin{array}{l}\text { Escritura - Preparación del } \\
\text { borrador original }\end{array}$ & Rocío Rodríguez Rico \\
\hline $\begin{array}{l}\text { Escritura - Revisión y } \\
\text { edición }\end{array}$ & $\begin{array}{l}\text { Rocío Rodríguez Rico (50\%) y Miguel Ángel Samano Rentería } \\
(50 \%)\end{array}$ \\
\hline Visualización & $\begin{array}{l}\text { Rocío Rodríguez Rico (50\%) y Miguel Ángel Samano Rentería } \\
\text { (50\%) }\end{array}$ \\
\hline Supervisión & Miguel Ángel Samano Rentería \\
\hline Administración de Proyectos & Rocío Rodríguez Rico \\
\hline Adquisición de fondos & Rocío Rodríguez Rico \\
\hline
\end{tabular}

